\title{
Long noncoding RNA PVT1-214 promotes proliferation and invasion of colorectal cancer by stabilizing Lin28 and interacting with miR-128
}

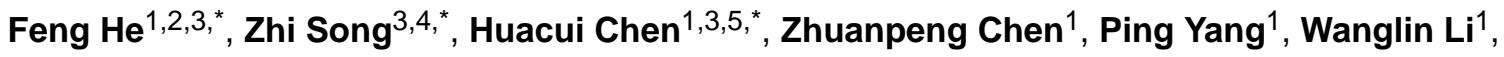 \\ Zhi Yang ${ }^{1}$, Tong Zhang ${ }^{1}$, Fei Wang ${ }^{1}$, Jianchang Wei ${ }^{1}$, Fang Wei ${ }^{1}$, Qiang Wang ${ }^{1}$, and Jie \\ Cao ${ }^{1,5, \#}$ \\ ${ }^{1}$ Department of General Surgery, Guangzhou Digestive Disease Center, Guangzhou First \\ People's Hospital, the Second Affiliated Hospital of South China University of Technology, \\ Guangzhou 510180, China \\ 2Department of Nephrology, Guangzhou First People's Hospital, the Second Affiliated Hospital of \\ South China University of Technology, Guangzhou 510180, China \\ ${ }^{3}$ Department of Pathology, Yale University School of Medicine, New Haven, 06520, Connecticut, \\ USA \\ ${ }^{4}$ Department of Operative Dentistry and Endodontics, Guanghua School of Stomatology, Hospital \\ of Stomatology, Sun Yat-sen University and Guangdong Provincial Key Laboratory of \\ Stomatology, Guangzhou, 510055, China \\ ${ }^{5}$ Department of General Surgery, Guangzhou First People's Hospital, Guangzhou Medical \\ University, Guangzhou 510180, China
}

\begin{abstract}
Long noncoding RNAs (lncRNAs) are implicated in human cancer, but their mechanisms of action are largely unknown. In this study, we investigated IncRNA alterations that contribute to colorectal cancer (CRC) through microarray expression profiling in CRC patient samples. Here, we report that the CRC-associated lncRNA PVT1-214 is a key regulator of CRC development and progression; patients with high $P V T 1-214$ expression had a shorter survival and poorer prognosis. In vitro and in vivo investigation of the role of $P V T 1-214$ revealed a complex integrated phenotype affecting cell growth, stem-like properties, migration, and invasion. Furthermore, using RNA pulldown and mass spectrometry, we found that Lin28 (also known as Lin28A), a highly conserved RNA-binding protein, is associated with PVT1-214. Strikingly, we found that PVT1-214 not only
\end{abstract}

\footnotetext{
Users may view, print, copy, and download text and data-mine the content in such documents, for the purposes of academic research, subject always to the full Conditions of use: http://www.nature.com/authors/editorial_policies/license.html\#terms

${ }^{\#}$ Correspondence should be addressed to: Department of General Surgery, Guangzhou Digestive Disease Center, Guangzhou First People's Hospital, the Second Affiliated Hospital of South China University of Technology, Guangzhou 510180, China. Tel: +862081048188, Fax: +862081048112, czhongt@126.com.

* Feng He, Zhi Song, and Huacui Chen contributed equally to this study.

Accession numbers: Microarray data have been deposited in the Gene Expression Omnibus database (accession number GSE109454).

Conflict of interest

The authors declare no conflict of interest.
} 
upregulated Lin 28 protein expression in CRC cells by stabilizing Lin28, but also participated in crosstalk with Lin 28 mRNA through competition for miR-128 binding, imposing an additional level of post-transcriptional regulation. In addition, we further show that PVT1-214 repressed expression of let-7 family miRNAs, which was abrogated by Lin2 8 knockdown. Taken together, our findings support a model in which the PVT1-214/Lin28/let-7 axis serves as a critical regulator of CRC pathogenesis, which may simulate a new direction for CRC therapeutic development.

\section{Keywords}

Colorectal cancer (CRC); LncRNA-PVT1-214; Lin28; Proliferation; Invasion

\section{INTRODUCTION}

Human genome sequence data indicate more than $90 \%$ of the genome is transcribed into RNA, but only $2 \%$ of these transcripts encodes proteins, whereas the vast majority are referred to as noncoding RNAs (ncRNA) ${ }^{1,2}$. Based on the RNA length, ncRNAs are conventionally divided into small ncRNAs (less than 200 nucleotides [nt]) and long ncRNAs (greater than $200 \mathrm{nt}$ ). Small ncRNAs, such as miRNAs, have been extensively studied, and their roles involved in multiple signal pathways contributing to gene regulation and cell function are well characterized in various cancer types ${ }^{3}$. Recently, IncRNAs have been implicated in control of cellular proliferation, apoptosis, differentiation, migration and/or invasion, and their dysregulated expression occurs in multiple human cancers ${ }^{4}$. LncRNAs are key mediators of tumorigenesis and progression by regulating oncogenic and tumorsuppressive pathways ${ }^{5,6}$. With recent advances in genomic sequencing, numerous novel transcripts are being discovered and stimulated the debate as to the functional transcripts. The ENCODE project suggests that annotated lncRNAs usually exhibit 2-40 isoforms per lncRNA locus ${ }^{7}$; however, it still remains an unresolved question to whether the splicing of lncRNAs is biologically meaningful. Recent studies indicate that RNA splicing isoforms are relevant to lncRNA functionality and regulation ${ }^{8,9}$.

In this study, we performed a comprehensive analysis to assess the differences between lncRNAs expression profiles in colorectal cancer (CRC), compared to paired adjacent nontumor tissues using lncRNA microarray. Overall, we observed 1085 upregulated IncRNAs and 1161 down-regulated IncRNAs in CRC samples. Among these, there were 14 PVT1 lncRNA transcripts. Intriguingly, the transcript PVT1-214 (Ensembl ID: ENST00000522875; 922 bp) was remarkably increased in human CRC tissues. PVT1 is located on chromosome 8q24.21, and its expression is upregulated in several cancers, including gastric, liver, lung, esophageal, colorectal, and osteosarcoma ${ }^{10-16}$, and its targets include FOXM1 (13), NOP2 (15), EZH2 (16), and c-MYC ${ }^{17}$. Additionally, Yan and colleagues ${ }^{18}$ showed that SOX2 binds to the $P V T 1$ promoter and enhances its transcription. Identification of the upstream and downstream targets of $P V T 1$ and the molecular mechanisms underlying its oncogenic functions and those of its transcripts will help elucidate its critical role in tumorigenesis. Although Takahashi and Guo reported that PVT1 serves as an oncogene in CRC cells ${ }^{15,19}$, the molecular mechanisms and downstream 
effector pathways of PVT1-214 in CRC cells are still unclear. Therefore, in this study, we characterized the pathologic relevance of $P V T 1-214$ in CRC pathogenesis.

\section{RESULTS}

\section{PVT1-214 is upregulated in human CRC tissues}

Given the role of lncRNAs and mRNAs in tumor pathogenesis, we set out to identify transcripts that potentially drive colorectal tumorigenesis. To do this, we performed microarray analysis on matched colorectal tumor and non-tumor samples from 6 CRC patients, and determined their lncRNA and mRNA expression profiles. Hierarchical clustering identified systematic variations in the expression of lncRNAs between CRC tissues and matched adjacent non-tumorous tissues (Figure 1a; Supplementary Figure S1; Supplementary Table S1). Microarray data were archived in NCBI Gene Expression Omnibus (GEO) and are accessible via GEO accession number GSE109454 (https:// www.ncbi.nlm.nih.gov/geo/query/acc.cgi?acc=GSE109454). We focused mainly on the upregulated lncRNAs because they can be readily as early diagnosis markers or therapeutic targets, compared to the down-regulated lncRNAs. In particular, we found that the 14 PVT1 transcripts were remarkably increased in CRC tissues (Supplementary Table S2). To validate our microarray findings, 11 transcripts with sequence lengths more than $500 \mathrm{nt}$ were analyzed by qRT-PCR in 6 pairs of CRC tissues and corresponding non-tumor tissues from cohort 1 (Supplementary Figure S2a). qRT-PCR analysis confirmed the microarray findings, demonstrating that the $11 P V T 1$ transcripts were differentially upregulated in CRC. Importantly, expression of the transcript PVT1-214 (Ensembl ID: ENST00000522875; 922 bp) was uniformly increased more than five times, compared with non-tumor tissues $(P<$ 0.0001; Supplementary Figure S2a), implying that $P V T 1-214$ may be associated with tumorigenesis or progression of CRC. Thus, we focused on this PVT1-214 transcript (human chromosome 8; 129001506-129108902; sequence listed in Supplementary Figure $\mathrm{S} 2 \mathrm{~b}$ ), and explored its expression pattern, clinical significance, and biological function in CRC.

To confirm the role of $P V T 1-214$ in CRC, we examined 45 paired CRC/non-tumor tissue specimens (Supplementary Table S3, cohort 1) and found that $P V T 1-214$ levels were significantly higher in CRC tissues $(P<0.001$; Figure 1b). Next, receiver operating characteristic (ROC) curve was performed to assess the sensitivity and specificity of PVT1-214 expression in differentiating CRC from normal tissues. Notably, PVT1-214 showed remarkable predictive significance, with an area under the ROC curve of 0.803 [95\% CI, 0.705-0.902; $P=0.001$; Figure 1c]. Additionally, ISH showed that $P V T 1-214$ was expressed in both the cytoplasm and nucleus of CRC tumor cells, but not in adjacent normal tissue (Figure 1d and e). Accordingly, these results strongly indicate that PVT1-214 is upregulated in CRC.

\section{PVT1-214 expression is associated with CRC progression and poor prognosis}

To evaluate whether PVT1-214 expression level is correlated with CRC progression, we investigated the association between $P V T 1-214$ and clinicopathological characteristics of CRC patients. We measured PVT1-214 expression in tumor samples from the second 
independent cohort including $92 \mathrm{CRC}$ patients (Supplementary Table S4, cohort 2) by qRTPCR. According to the median ratio of relative PVT1-214 expression, patients were divided into two groups: the high group was defined by a PVT1-214 expression ratio $\geq$ median ratio, and the low group was defined by a $P V T 1-214$ expression ratio < median ratio. As illustrated in Table 1, greater $P V T 1-214$ expression was positively associated with tumor size $(P=0.029)$, distant metastasis $(P=0.020)$, and TNM stage, according to the American Joint Committee on Cancer (AJCC) staging system $(P=0.030)$. Moreover, kaplan-Meier analysis and log-rank test showed a positive correlation between tumor-associated $P V T 1-214$ expression, and reduced overall survival $(P=0.010$; Figure 1f) and recurrencefree survival $(P=0.006$; Figure $1 \mathrm{~g})$. Additionally, multivariate analysis showed that $P V T 1-214$ expression (95\% CI, 1.579-5.186; $P=0.008$ ) and distant metastasis (95\% CI, 1.211-4.032; $P=0.022$ ) were independent prognostic factors for overall survival in CRC (Supplementary Table S5).

\section{PVT1-214 promotes CRC cell proliferation, stem-like properties, migration, and invasion in vitro}

To confirm the biological effects of $P V T 1-214$ on CRC development, we first measured the baseline levels of $P V T 1-214$ in 5 CRC cells lines and a normal human colonic epithelial cell line, NCM460. We found that PVT1-214 expression was significantly elevated in CRC cells lines compared to NCM460 cells (Supplementary Figure S3a). Additionally, we observed that PVT1-214 in SW480 and HCT116 cells is distributed in both the cytoplasm and nucleus, but is expressed more highly in the cytoplasm (Supplementary Figure S3b). Next, we constructed CRC cell lines with either stable PVT1-214 overexpression, or PVT1-214 knockdown (Figure 2a; Supplementary Figure S4a). We found that compared to the paralleled stable cell lines with the empty vector, exogenous expression of PVT1-214 accelerated the proliferative capacity of SW480 and HCT116 cells (Figure 2b). Consistent with the phenotype of increased cell proliferation, immunofluorescent staining for 5ethynyl-2'-deoxyuridine (EdU) incorporation confirmed that SW480-PVT1-214 and HCT116-PVT1-214 cell populations contained more EdU-positive cells with new DNA synthesis, $51.2 \%$ and $42.7 \%$, respectively, compared with the control cell populations (30.8\% and $27.3 \%$, respectively; Figure $2 \mathrm{c}$ and d). Next, we performed fluorescenceactivated cell sorting (FACS) to detect cellular DNA content changes during cell cycle. We found that both PVT1-214-overexpressing SW480 and HCT116 cells displayed relatively decreased proportions of cells in G1-phase, but had a significant increase in the percentage of cells in S-phase (Figure 2e and f). Conversely, knockdown of PVT1-214 suppressed CRC cell proliferation (Supplementary Figure S4b), decreased EdU staining (Supplementary Figure S4c and d), and reduced proportions of cells in S-phase (Supplementary Figure S4e and f). Together, these findings show that PVT1-214 exerts a role in stimulating CRC cell proliferation.

In attempt to understand the function of $P V T 1-214$ in tumor initiation, we carried out in vitro sphere formation assays using SW480 cells and found that $P V T 1-214$ overexpression promotes faster spheroid formation, suggesting an increased stem-like potential compared with control cells. SW480-PVT1-214 cells formed spheres within 7 days in serum-free media, and the full spherical morphology was achieved within 14 days. In contrast, control 
SW480 cells took 21 days to generate spheroids (Figure 2g). To investigate whether PVT1-214 plays a role in metastasis, we performed Transwell migration assays, and found that up-regulation of PVT1-214 significantly enhanced the migratory (Figure $2 \mathrm{~h}$ and i) and invasive capacity (Figure $2 \mathrm{j}$ and k) of SW480 and HCT116 cells, whereas PVT1-214 knockdown abolished the aforementioned effects (Supplementary Figure S4g-j). Thus, these results suggest that $P V T 1-214$ promotes CRC initiation and metastasis in vitro.

\section{PVT1-214 induces tumorigenesis and metastasis of CRC cells in vivo}

To evaluate the effects of $P V T 1-214$ on tumorigenesis in vivo, we injected HCT116 cells expressing either $P V T 1-214$ or empty vector subcutaneously into nude mice for xenograft implantation. We found that $P V T 1-214$ overexpressing xenografts grew faster than control xenograft tumors and were significantly larger, demonstrated by increased mean tumor weight and volume (Figure 3a-c). Moreover, PVT1-214-expressing xenograft tumors displayed increased Ki67 and cyclinD1 expression, indicating these tumors were more proliferative than control tumors (Figure 3d). To investigate the effect of PVT1-214 on CRC liver metastases, we used an in vivo spleen-hepatic metastasis model, whereby $5 \times 10^{6}$ PVT1-214-overexpressing and control HCT116 cells were injected into the spleen of $\mathrm{BALB} / \mathrm{c}$-nude mice and metastasis to the liver was evaluated using bioluminescence imaging. Five weeks after injection, we found that PVT1-214 overexpression significantly enhanced hepatic metastasis, confirmed by bioluminescence imaging (Figure $3 \mathrm{e}$ ) and quantification of liver surface metastatic foci (Figure 3f). In summary, we suggest that PVT1-214 is a cellular proto-oncogene that promotes CRC development and metastases.

\section{PVT1-214 binds Lin28 and stabilizes its expression}

Several recent studies have indicated that many lncRNAs regulate signal pathways via interacting with specific proteins ${ }^{10,12}$. To determine whether $P V T 1-214$ affects the behavior of CRC cells by this way, we sought to identify proteins associated with $P V T 1-214$ by RNA pull-down assay. RNA-associated proteins were analyzed by SDS-PAGE and silver staining (Figure 4a), and the binding band specific to the PVT1-214 pull-down were excised and subjected to mass spectrometry (Supplementary Table S6). Interestingly, Lin28 was identified from three independent RNA pull-down assays by western blotting in SW480 cell extracts (Figure 4b). We next performed RIP with an antibody against Lin28 using cell extracts from SW480 cells, and observed enrichment of $P V T 1-214$ pull-down (Figure 4c). Notably, deletion-mapping analyses identified that the $3^{\prime}$-end (672-922 nt) of PVT1-214 is required for association with Lin 28 (Figure 4d). Finally, we analyzed the interaction between PVT1-214 and Lin28 using RIP in cytoplasmic and nuclear fractions, and found that PVT1-214 and Lin28 interaction occurs in the cytoplasmic fraction (Supplementary Figure S5a). Therefore, these findings indicated the specific association between PVT1-214 and $\operatorname{Lin} 28$.

Next, we attempted to determine the functional relevance for associations between PVT1-214 and Lin28. We detected significant up-regulation of Lin28 in PVT1-214 overexpressing SW480 and HCT116 cells, while PVT1-214 knockdown resulted in downregulation of Lin28 (Figure 4e). Importantly, we did not observe a significant change in Lin28 mRNA levels (Supplementary Figure S5b). Similar results were observed in 
PVT1-214-overexpressig xenograft tumors by western blot and IHC analysis (Supplementary Figure S5c and d). Additionally, no differences in Lin 28 mRNA expression were observed in vivo (Supplementary Figure S5e). Then, we hypothesize that PVT1-214 binds to Lin28 and exerts function at the posttranscriptional or translational level. To investigate these hypotheses, we found that, compared to DMSO-treated control cells, Lin28 was decreased by the protein synthesis inhibitor cycloheximide (CHX) and increased by the proteasome inhibitor MG-132 (Figure 4f). We next treated PVT1-214-overexpressing SW480 cells with CHX or MG-132, and found that only MG-132 abolished the changes in Lin28 protein levels in PVT1-214-overexpressing cells (Figure 4g), suggesting that Lin28 degradation occurs via the ubiquitin-proteasome pathway. Together, these data suggest that PVT1-214 reduces Lin28 protein degradation and enhances its stability in CRC cells.

\section{PVT1-214 acts as a molecular sponge for miR-128 and controls the miR-128 target, Lin28}

Concerning epigenetic regulation of targets in the nucleus, emerging studies have demonstrated that certain lncRNAs can act as competing endogenous RNAs (ceRNA), functioning as microRNA sponges to regulate expression of target genes in the cytoplasm. Several studies have identified that $P V T 1$ might act as a ceRNA and sponge for miR-152 ${ }^{20}$, miR-195 ${ }^{16}$, or miR-203 ${ }^{14}$. Interestingly, bioinformatics analysis of miRNAs target recognition sequences on $P V T 1-214$ and the $3^{\prime}$-UTR of $\operatorname{Lin} 28$ revealed that miR-128 is complementary to $P V T 1-214$ sequence, as well as to the Lin283'-UTR (Figure 5a). To confirm this finding, we cloned the PVT1-214 cDNA downstream of the luciferase gene (pGL3-PVT1-214-wt), and transfected it together with miR-128, or a negative control. We found that when miR-128 was overexpressed, luciferase activity was reduced by $39 \%$, compared to the control of empty vector (Figure $5 b$ ). To further determine whether the luciferase activity decrease expression from the pGL3-PVT1-214-wt vector was associated with direct interaction between miR-128 and PVT1-214 putative binding site, we mutated the miR-128 binding site by site-directed mutagenesis, generating a pGL3-PVT1-214-mut vector. As expected, point mutations in the tentative PVT1-214 abrogated the repressive effect of miR-128 (Figure 5b).

The core component of miRNA ribonucleoprotein complexes (miRNPs) silencing complexes in the cytoplasm, present in anti-Ago2 immunoprecipitates to form RNA-induced silencing complexes (RISCs), generally containing miRNAs and their interacting RNAcomponents ${ }^{21,22}$. To test whether PVT1-214 associates with miRNPs, we performed RIP experiments using anti-Ago2 in SW480 cell extracts. We found that PVT1-214 and miR-128 were preferentially enriched (262-fold; 255-fold) in Ago2-containing miRNPs compared to anti-IgG immunoprecipitates. RIP primers against human $F O S$ verified successfully the Ago2-associated RNA immunoprecipitation by qRT-PCR (Figure 5c, top panel). Besides, the positive control for the RIP procedure showed that $U 1$ snRNA was enriched at significant level in anti-SNRNP70 than anti-IgG immunoprecipitates (Figure 5c, bottom panel). Thus, our results demonstrate that PVT1-214 is present in Ago2-containing miRNPs, likely through interaction with miR-128, consistent with our bioinformatic analysis and luciferase activity results. 
Next, to test whether miR-128 targets the Lin $283^{\prime}$-UTR, we performed western blotting for Lin28 in the presence of miR-128 mimics or inhibitor. We observed decreased Lin28 expression when SW480 and HCT116 cells were treated with the miR-128 mimics, indicating that miR-128 downregulates Lin28 protein expression (Supplementary Figure S6a). To confirm this mechanism, we cloned the human Lin283'-UTR into the pGL3luciferase reporter vector, and constructed two miR-128 binding mutants in which the putative miR-128 binding sites ACUGU, CACUGU in the Lin283'-UTR were mutated into UGACA, GUGACA, respectively (Supplementary Figure S6b, top panel). As expected, double luciferase report results showed miR-128 mimics resulted in significant repression of Lin28 transcript levels, however, point mutations in the Lin283'-UTR abrogated the repressive effect of miR-128 (Supplementary Figure S6b, bottom panel). Collectively, these results establish that Lin28 is a target of miR-128.

Furthermore, to investigate whether $P V T 1-214$ regulates Lin28 expression by sponging miR-128, we detected the level of miR-128 in PVT1-214 or PVT1-214 mutation of the putative miRNA recognition element (MRE) overexpressed-HCT116 cells, and found ectopically expressed PVT1-214, but not the mutant, significantly sequestered the miR-128 expression (Figure 5d). Next, to validate whether PVT1-214 can regulate Lin28 expression via competition for miR-128 binding, as shown in Figure 5e, $P V T 1-214$ significantly increased Lin 28 protein expression, however, mutagenesis of the miR-128 recognition element in PVT1-214 abrogated the function effectively. Conversely, inhibition of miR-128 overcame the suppression of Lin 28 by PVT1-214 knockdown (Figure 5f). Moreover, we also evaluated the effect of $P V T 1-214$ expression on endogenous Lin28 with or without exogenous miR-128 expression, and found that $P V T 1-214$ overexpression significantly rescued the silencing effect of miR-128 on Lin 28 protein expression (Figure $5 \mathrm{~g}$ ). In human CRC samples $(\mathrm{n}=45)$, we also observed a statistically significant inverse correlation between PVT1-214 and miR-128 transcript levels (two-sided Pearson correlation, $\mathrm{r}=$ $-0.527, P=0.001$, Figure $5 \mathrm{~h})$. Together, these data indicate that by interacting with miR-128, $P V T 1-214$ also acts as a molecular sponge for the miR-128 target Lin28, to partly modulate the derepression of Lin28 in post-transcriptional level.

\section{PVT1-214 represses let-7 family miRNAs expression through Lin28 upregulation}

The RNA binding protein Lin28 and Lin28B specifically binds to the Let-7 miRNAs loop region to inhibit their biogenesis through preventing both Drosha- and Dicer-mediated cleavage, then accelerates the turnover of let- 7 precursors ${ }^{23,24}$. Consistent with this, expression of let-7a and let-7f were dramatically decreased when Lin 28 was overexpressed (Supplementary Figure S7a); conversely, Lin28 knockdown increased let-7a and let-7f expression (Supplementary Figure S7b). However, these primary transcripts were not induced in Lin28 knockdown cells (Supplementary Figure S7c). Therefore, we speculated that PVT1-214 may inhibit let-7 family members through up-regulation of Lin28. To determine this, we examined mature miRNA levels of individual let-7 family members in SW480 cells stably overexpressing PVT1-214. As expected, let-7a, let-7b, let-7d, let-7f, let-7g, and let-7i were dramatically repressed in cells overexpressing PVT1-214 (Figure 6a). We next examined whether up-regulation of Lin28 is necessary for PVT1-214 repression of 
let-7 miRNAs, and we found that Lin28 knockdown could effectively abrogate PVT1-214 induced let-7 repression (Figure 6b).

We further investigated expression of Lin28 in CRC using IHC. Interestingly, we found that Lin28 was strongly expressed in high clinical stage CRC samples, and was slightly downregulated in low clinical stage samples (Figure 6c). Immunoblotting analysis showed that Lin28 expression was significantly upregulated in eight human CRC samples, compared to adjacent non-tumor tissues (Figure 6d). Moreover, when we compared expression of PVT1-214 and let-7a in 45 CRC specimens, we found significant negative association between the two groups (two-sided Pearson correlation, $\mathrm{r}=-0.539, P<0.001$, Figure 6e). Together, these findings indicate that up-regulation of Lin28 is necessary for PVT1-214 to down-regulate let-7 family members through post-transcriptional regulation.

\section{PVT1-214 facilitates CRC cell proliferation and metastasis in a Lin28-mediated manner}

To investigate whether PVT1-214 functions in a Lin28-mediated manner in CRC, we first performed immunoblotting analysis in HCT116 cells overexpressing PVT1-214, and SW480 cells with PVT1-214 knockdown. We found that when PVT1-214 was overexpressed, Lin28, and well-characterized CRC proliferation genes, such as c-myc, c-Met and Cyclin D1, were upregulated. However, the opposite was true in PVT1-214 knockdown cells, where these proteins were decreased. Interestingly, we observed that $\mathrm{p} 21$ expression had the inverse response (Figure 7a). Moreover, we found that in PVT1-214-overexpressing cells, the epithelial marker E-cadherin was down-regulated, and the mesenchymal marker vimentin was upregulated; conversely, the opposite was true in PVT1-214 knockdown cells (Figure 7a). Therefore, these results suggest that PVT1-214 exerts an important role in promoting CRC proliferation and an epithelial-to-mesenchymal response. Furthermore, an EdU assay indicated that PVT1-214 overexpression promoted SW480 cell proliferation, which was impaired by Lin 28 knockdown (Figure 7b, c). Transwell assays also showed that Lin28 knockdown partially abrogated the effect of PVT1-214 overexpression on CRC cell metastasis, and partially attenuated sphere-forming capacity of SW480 cells (Figure 7b, c). Together, our results indicate that $P V T 1-214$ promotes CRC cell proliferation and metastasis in a Lin28-mediated manner.

\section{DISCUSSION}

Recently, vast numbers of lncRNA transcripts were discovered through cDNA library sequencing and microarray analysis. However, although these techniques quantify the presence of these transcripts, they do not provide information about their biological activity 25. In this study, we identified that the transcript of PVT1 (PVT1-214; Ensembl ID: ENST00000522875) was increased in human CRC tissues (Figure 1A and B). We further validated PVT1-214 expression in CRC clinical samples and determined that increased PVT1-214 expression was associated with advanced pathologic stage, tumor size, and distant metastasis. Importantly, PVT1-214 overexpression was associated with poor prognosis and could be an independent prognostic indicator. Moreover, by applying lossand gain-of-function approaches, we identified that PVT1-214 markedly promoted CRC cell proliferation, stem-like properties, and metastasis, both in vitro and in vivo. Full-length 
$P V T 1$ is an oncogene ${ }^{10,12,17}$, and here, we reveal for the first time that the PVT1-214 transcript may also be an oncogene in CRC and play a crucial role in CRC pathogenesis. Furthermore, we found that PVT1-214 was distributed in the cytoplasm and nucleus in CRC cells, and directly bound Lin28, a highly conserved RNA-binding oncoprotein that promotes cancer progression ${ }^{26,27}$. We also indicated that PVT1-214 increased Lin28 expression in CRC cells, suggesting that Lin28 could be an important underlying regulator of PVT1-214 function.

Our results revealed that $P V T 1-214$ was associated with Lin28 and enhanced its stability through the ubiquitin-proteasome pathway. However, the underlying mechanisms of how PVT1-214 regulates ubiquitination and/or ubiquitination-associated enzymes deserved further investigation. Wang et al. reported that Lin28 expression is elevated in CRC, and promotes the transition from S-phase to G2/M-phase, stimulating proliferation ${ }^{28}$. Similarly, we observed up-regulation of Lin28 in advanced stage CRC tissues, which was associated with high PVT1-214 expression. Notably, we found that Lin28 knockdown abrogated PVT1-214-mediated CRC cell proliferation and metastasis, indicating that PVT1-214 functions in a Lin28-dependent manner.

Recent researches show that ncRNAs function as microRNA sponges to reduce the amount of miRNAs available to target messenger RNAs (mRNAs) ${ }^{29,30}$. Inspired by the discoveries of the interaction between IncRNAs and miRNAs ${ }^{31}$, we confirmed the existence of crosstalk between PVT1-214 and Lin28 mRNA through competition for miR-128 binding. Consistent with PVT1-214 sequestration of miR-128, we found that $P V T 1-214$ overexpression significantly rescued the silencing effect of miR-128 on Lin 28 protein expression. In addition, $\mathrm{Lu}$ and colleagues ${ }^{32}$ reported that miR-128 was differentially reduced in CRC tissues, and patients with lower miR-128 expression had shorter survival. Additionally, upregulation of miR-128 expression promoted the chemo-sensitivity of CRC cells, and suppressed the potential invasiveness, suggesting miR-128 downregulation associated with CRC progression. Concordantly, we demonstrated that PVT1-214 levels were inversely correlated with miR-128 expression in CRC specimens, and mutagenesis of the miR-128 recognition element in $P V T 1-214$ partly abrogated its effect on upregulating Lin28 protein expression in CRC cells, indicating that PVT1-214 modulates the de-repression of Lin28 by interacting with miR-128. Concurrently, this additional level of post-transcriptional regulation demonstrated that $P V T 1-214$ also acted as a molecular sponge for miR-128 and thus regulating Lin28 expression.

The let-7 family is one of the most evolutionarily conserved miRNA families, and multiple let-7 isoforms are required for development and tumor suppressor function ${ }^{33}$. RNA-binding proteins Lin28 and Lin28B are important regulators of let-7 miRNAs expression via posttranscriptional inhibition of let-7 biogenesis, and in turn, Lin28 and Lin28B are targeted by let- ${ }^{34}$. Importantly, we show that $P V T 1-214$ overexpression repressed expression of let-7 family miRNAs, and Lin28 knockdown abrogated PVT1-214-mediated let-7 repression. Interestingly, we observed a significant inverse correlation between PVT1-214 and let-7a expression in CRC tissues (Figure 6E). Consequently, we show a critical role of PVT1-214 on regulating the Lin28/let-7 axis in CRC proliferation and progression. 
Although $P V T 1$ acting as an oncogene in several cancers has been well documented, characterizations of the $P V T 1$ isoforms is still not be reported. Based on our microarray data, $14 P V T 1$ transcripts were identified in CRC tissues. Considering these factors that validation results of qRT-PCR analysis, independent amplification and pre-experimental function determination, we selected to characterize the PVT1-214 transcript and showed PVT1-214/Lin28/let-7 axis served as an important regulator of CRC pathogenesis. Because different transcripts appear to be largely different, including the functions and expression trends, the characterizations of other $P V T 1$ transcripts in cancers require future researches. Cho et al. showed mutant alleles of the $P V T 1$ promoter were significantly enriched up to 3fold expression and conferred a distinct growth advantage of MDA-MD-231 cells, moreover, cancer genome sequencing identified recurrent mutations encompassing the human PVT1 promoter ${ }^{35}$. Of note, $14 P V T 1$ transcripts are uniformly up-regulated in CRC, which may be related to the genome editing in $P V T 1$ promoter in CRC. Additionally, $P V T 1-214$ is a valuable transcript of $P V T 1$, it may be relevant to the initiation and progression in other cancers, its exact roles and its regulatory mechanisms (transcriptional regulation, epigenetic modification, mutations in PVT1 promoter, and so on) deserve further investigations, which may be important targets for tumors diagnosis and therapy.

Collectively, we show that up-regulation of PVT1-214 acts as an oncogenic driver in CRC, which is mediated by stabilization and post-transcriptional regulation of Lin28, targeting the Lin28/let-7 axis to promote tumorigenesis (Figure 7D). Our study may therefore provide the basis for a novel therapeutic strategy to target the PVT1-214/Lin28/let-7 axis in CRC.

\section{MATERIALS AND METHODS}

\section{Microarrays and computational analysis}

Microarray analysis was performed by KangChen Bio-tech, Shanghai, PR China. Fresh colorectal tumor samples and matched non-tumor samples from 6 CRC patients (Supplementary Table S1) were selected randomly for microarray analysis. The assay started with a 4- $\mu$ g total RNA sample. Complementary DNA was synthesized, labeled, purified, and hybridized to the microarray (Arraystar). Fluorescence images were obtained by a laser scanner and digitized using Affymetrix Transcriptome Analysis Software. Raw data matrix is analyzed by first subtracting the background matrix and then normalization. A 2-sample $t$ test was used to statistical analysis. Microarray data of this study can be accessed via Gene Expression Omnibus (GEO) GSE109454.

\section{Patients and clinical samples}

The use of human samples in this study was sanctioned by the local ethics committee at the Guangzhou First People's Hospital, Guangzhou Medical University (Guangzhou, China). Two independent cohorts involving a total of 137 CRC patients were enrolled. Forty-five fresh CRC tissue pairs were collected from patients of Guangzhou First People's Hospital from January 2012-August 2013. Another 92 CRC tissue pairs were collected from the same hospital from August 2009-September 2014 and used for further validation and analysis. All these fresh tissues were collected and processed immediately within 15 minutes in the operating room. De-identified patient clinical information was showed in Supplementary 
Tables S2 and S3. None of the patients received preoperative treatment. Normal adjacent non-tumor tissues were collected by a distance of at least $5 \mathrm{~cm}$ from the tumor.

\section{Cell culture}

CRC cell lines (LOVO, SW480, HCT116, SW620, and HT29) (ATCC) were cultured in RPMI-1640 medium (Gibco). The human colonic epithelial cell line NCM460 was maintained in Dulbecco's modified Eagle's medium (Gibco). Cells were cultured in serumcontaining medium in a humidified $5 \% \mathrm{CO}_{2}$ atmosphere at $37^{\circ} \mathrm{C}$.

\section{qRT-PCR and subcellular fractionation}

Quantification expression of $P V T 1-214$ and other transcripts and genes were detected by qRT-PCR, according to the manufacturer's instructions (Bio-Rad). qRT-PCR was carried out by the Applied Biosystems 7500 Sequence Detection system. Three replicates were used for each sample. Primer sequences are showed in Supplementary Table S7. Primers for miRNAs were designed and synthesized by Guangzhou RiboBio (RiboBio, China).

Cytosolic and nuclear fractions were isolated and collected with the Nuclear/Cytoplasmic Isolation Kit (Biovision) according to the manufacturer's instructions. qRT-PCR was applied to analyzed RNA levels of a nuclear control transcript ( $R N U 6-1)$, a cytoplasmic control transcript (GAPDH), and PVT1-214 in the fractions.

\section{Plasmid construction, lentiviral construction, and cell transfections}

Details of the procedure were provided in the Supplementary Materials and Methods.

\section{RNA pull-down assay and mass spectrometry}

Plasmids containing PVT1-214 and an antisense sequence were linearly cut, transcribed, and biotin-labeled in vitro with Bio-16-UTP (Life Technologies) using a MAXIscript T7 Transcription Kit (Life Technologies). Protein-RNA interactions were determine using a Pierce Magnetic RNA-Protein Pull-Down Kit (Life Technologies) with SW480 cell lysates. The retrieved proteins were detected by western blot analysis or resolved by in-gradient gel electrophoresis, followed by mass spectrometry identification.

\section{In vitro colonosphere formation, cell migration, and invasion assays}

SW480 colonospheres were generated as previously described ${ }^{36}$. Detailed description of cell migration and invasion assays were available in the Supplementary Materials and Methods.

\section{Bioinformatics prediction and dual-luciferase reporter assays}

The potential miR-128-binding sites of PVT1-214 sequence predicted by computer-based algorithms was available at http://www.microrna.org. The PVT1-214 full-length sequence (wild type, wt) and the PVT1-214 mutant (only mutated the putative miR-128 binding sites) were synthesized through DNA synthesis technology and then cloned in the pGL3 luciferase vector (Addgene) respectively. 
MiR-128 target genes were predicted using TargetScan (http://www.targetscan.org) and Pictar (http://pictar.mdc-berlin.de/). To assess whether miR-128 regulates directly Lin28, we constructed wild-type and mutated Lin28-3'-UTR (Lin28-wt / Lin28-Mut) luciferase reporter vectors in the pGL3 luciferase vector. Luciferase and renilla signals were measured using the Dual Luciferase Reporter Assay Kit (Promega). Detected luciferase activity required to normalize to the renilla luciferase activity.

\section{Western blotting, immunohistochemistry (IHC), and in situ RNA hybridization (ISH)}

Western blotting and IHC were performed as previously described ${ }^{37}$. Antibody information is listed in Supplementary Table S8. ISH assay was carried out as previously described ${ }^{38}$. A locked nucleic acid probe with complementarity to a section of PVT1-214 (5DigN/ TAACGGGCTCCCAGATTCACAAGC/3 DigN-custom LNA detection probe) was labeled with $5^{\prime}$-digoxigenin and $3^{\prime}$-digoxigenin, and synthesized by Exiqon Company.

\section{RNA-binding protein immunoprecipitation (RIP) assay}

RIP assay was carried out using the EZ-Magna RIP kit (Millipore) according to the protocol provided by the manufacturer. Briefly, whole cell lysate was incubated with RIP buffer containing magnetic beads conjugated to a human anti-Lin28 antibody, human anti-Ago2 antibody, or negative control normal mouse IgG. Anti-SNRNP70 antibody was applied for a positive control. Samples lysis were all treated with Proteinase K, and the RNA immunoprecipitations were performed qRT-PCR assay to identify the presence in binding target antibody with respective primers.

\section{Xenograft tumor model}

BALB/c-nude mice (5-6 weeks of age) were purchased from the laboratory animal center of Sun Yat-sen University. Animals were reared in the barrier facilities on a 12-hrs light/dark cycle. The experimental procedures were approved by the Institutional Animal Care and Use Committee of our institution. For bioluminescent imaging, SW480-Luc-vector and SW480Luc- $P V T 1-214\left(5 \times 10^{6}\right.$ cells) were subcutaneously implanted in dorsal flank regions of $\mathrm{BALB} / \mathrm{c}-\mathrm{nu}$ mice ( $\mathrm{n}=5$ per group). Tumor growth was monitored weekly; length, width, and thickness were measured separately with calipers, and tumor volumes were determined with the equation $\left(\mathrm{L} \times \mathrm{W}^{2}\right) / 2$. After 5 weeks, tumor luminescence was detected by IVIS bioluminescence imaging system (Caliper Life Sciences). Injections of D-luciferin with 150 $\mathrm{mg} / \mathrm{kg}$, and images were acquired at $15 \mathrm{~min}$. The following general anesthesia, then tumors were excised, weighed, processed for paraffin embedding and stored in liquid nitrogen. Serial $5.0 \mu \mathrm{m}$ sections were used for IHC and hematoxylin and eosin (H\&E) staining (Sigma-Aldrich).

\section{Liver metastasis model}

In vivo liver metastasis was carried out as previously described ${ }^{37}$. Briefly, male BALB/cnude mice (6-8 weeks of age) were anesthetized, and the spleen was then exteriorized through a left lateral flank incision. SW480-Luc-vector and SW480-Luc-PVT1-214 cells $\left(5 \times 10^{6}\right)$ in $100 \mu$ of Hank's balanced salt solution were injected into the spleen parenchyma using a 25-gauge needle. Bioluminescence images were obtained by the IVIS 
bioluminescence imaging system (Caliper Life Sciences) on day 28. After general anesthesia, tumors and metastasis target organs were excised and used for further experiments. All experiments were approved by the relevant guidelines of Guangzhou First People's Hospital.

\section{Statistical analysis}

All statistical analyses were carried out using SPSS 17.0 statistical software package and GraphPad Prism 7.0. All data are showed with the mean \pm SEM. The Student's $t$ test and one-way analysis of variance (ANOVA) were used to compare two or more groups for statistical analysis, respectively. The nonparametric Mann-Whitney-Wilcoxon test was performed to compare the correlation between PVT1-214 level and clinical parameters. Survival curves were plotted by the Kaplan-Meier method and compared statistically using the log rank test. Univariate analysis and multivariate models were performed with the Cox proportional-hazards regression model. Risk score analysis was performed to evaluate the effectiveness of $P V T 1-214$ for prediction. A Pearson correlation coefficient analysis was used to assess the correlations. $P$ value $<0.05$ was considered statistically significant.

\section{Supplementary Material}

Refer to Web version on PubMed Central for supplementary material.

\section{Acknowledgments}

This work was supported by grants from the National Natural Science Foundation of China (Grant no. 81600654 and 81600862); the Guangdong Natural Science Foundation (Grant no. 2017A030311035, 2016A030313490 and 2016A030310227); the Fundamental Research Funds for the Central Universities (Grant no. XZYXD2175080); and the Guangdong Provincial Department of Science and Technology (Grant no. 2017A020215146).

\section{References}

1. Djebali S, Davis CA, Merkel A, Dobin A, Lassmann T, Mortazavi A, et al. Landscape of transcription in human cells. Nature. 2012; 489:101-108. [PubMed: 22955620]

2. Tay Y, Rinn J, Pandolfi PP. The multilayered complexity of ceRNA crosstalk and competition. Nature. 2014; 505:344-352. [PubMed: 24429633]

3. Rupaimoole R, Slack FJ. MicroRNA therapeutics: towards a new era for the management of cancer and otherdiseases. Nat Rev Drug Discov. 2017; 16:203-222. [PubMed: 28209991]

4. Batista PJ, Chang HY. Long noncoding RNAs: cellular address codes in development and disease. Cell. 2013; 152:1298-1307. [PubMed: 23498938]

5. Dhamija S, Diederichs S. From junk to master regulators of invasion: lncRNA functions in migration, EMTand metastasis. Int J Cancer. 2016; 139:269-280. [PubMed: 26875870]

6. Malone CD, Hannon GJ. Small RNAs as guardians of the genome. Cell. 2009; 136:656-668. [PubMed: 19239887]

7. Volders PJ, Helsens K, Wang X, Menten B, Martens L, Gevaert K, et al. LNCipedia: a database for annotated human lncRNA transcript sequences andstructures. Nucleic Acids Res. 2013; 41:D246D251. [PubMed: 23042674]

8. Chen FC, Pan CL, Lin HY. Functional Implications of RNA Splicing for Human Long Intergenic Noncoding RNAs. Evol Bioinform Online. 2014; 10:219-228. [PubMed: 25574121]

9. Cao C, Zhang T, Zhang D, Xie L, Zou X, Lei L, et al. The long non-coding RNA, SNHG6-003, functions as a competing endogenous RNA topromote the progression of hepatocellular carcinoma. Oncogene. 2017; 36:1112-1122. [PubMed: 27530352] 
10. Xu MD, Wang Y, Weng W, Wei P, Qi P, Zhang Q, et al. A Positive Feedback Loop of lncRNAPVT1 and FOXM1 Facilitates Gastric CancerGrowth and Invasion. Clin Cancer Res. 2017; 23:2071-2080. [PubMed: 27756785]

11. Kong R, Zhang EB, Yin DD, You LH, Xu TP, Chen WM, et al. Long noncoding RNA PVT1 indicates a poor prognosis of gastric cancer and promotescell proliferation through epigenetically regulating p15 and p16. Mol Cancer. 2015; 14:82-96. [PubMed: 25890171]

12. Wang F, Yuan JH, Wang SB, Yang F, Yuan SX, Ye C, et al. Oncofetal long noncoding RNA PVT1 promotes proliferation and stem cell-likeproperty of hepatocellular carcinoma cells by stabilizing NOP2. Hepatology. 2014; 60:1278-1290. [PubMed: 25043274]

13. Wan L, Sun M, Liu GJ, Wei CC, Zhang EB, Kong R, et al. Long Noncoding RNA PVT1 Promotes Non-Small Cell Lung Cancer Cell Proliferationthrough Epigenetically Regulating LATS2 Expression. Mol Cancer Ther. 2016; 15:1082-1094. [PubMed: 26908628]

14. Li PD, Hu JL, Ma C, Ma H, Yao J, Chen LL, et al. Upregulation of the long non-coding RNA PVT1 promotes esophageal squamous cellcarcinoma progression by acting as a molecular sponge of miR-203 and LASP1. Oncotarget. 2017; 8:34164-34176. [PubMed: 28404954]

15. Takahashi Y, Sawada G, Kurashige J, Uchi R, Matsumura T, Ueo H, et al. Amplification of PVT-1 is involved in poor prognosis via apoptosis inhibition in colorectal cancers. Br J Cancer. 2014; 110:164-171. [PubMed: 24196785]

16. Zhou Q, Chen F, Zhao J, Li B, Liang Y, Pan W, et al. Long non-coding RNA PVT1 promotes osteosarcoma development by acting as amolecular sponge to regulate miR-195. Oncotarget. 2016; 7:82620-82633. [PubMed: 27813492]

17. Tseng YY, Moriarity BS, Gong W, Akiyama R, Tiwari A, Kawakami H, et al. PVT1 dependence in cancer with MYC copy-number increase. Nature. 2014; 512:82-96. [PubMed: 25043044]

18. Wang Y, Zhou J, Wang Z, Wang P, Li S. Upregulation of SOX2 activated LncRNA PVT1 expression promotes breast cancer cellgrowth and invasion. Biochem Biophys Res Commun. 2017; 493:429-436. [PubMed: 28882595]

19. Guo K, Yao J, Yu Q, Li Z, Huang H, Cheng J, et al. The expression pattern of long non-coding RNA PVT1 in tumor tissues and inextracellular vesicles of colorectal cancer correlates with cancer progression. Tumour Biol. 2017; 39:9122-9132.

20. Zheng J, Yu F, Dong P, Wu L, Zhang Y, Hu Y, et al. Long non-coding RNA PVT1 activates hepatic stellate cells through competitivelybinding microRNA-152. Oncotarget. 2016; 7:62886-62897. [PubMed: 27588491]

21. Izaurralde E. Elucidating the temporal order of silencing. EMBO Rep. 2012; 13:662-673. [PubMed: 22722480]

22. Filipowicz W, Bhattacharyya SN, Sonenberg N. Mechanisms of post-transcriptional regulation by microRNAs: are the answers insight. Nat Rev Genet. 2008; 9:102-114. [PubMed: 18197166]

23. Heo I, Joo C, Cho J, Ha M, Han J, Kim VN. Lin28 mediates the terminal uridylation of let-7 precursor MicroRNA. Mol Cell. 2008; 32:276-284. [PubMed: 18951094]

24. Newman MA, Thomson JM, Hammond SM. Lin-28 interaction with the Let-7 precursor loop mediates regulated microRNAprocessing. RNA. 2008; 14:1539-1549. [PubMed: 18566191]

25. Mattick JS, Makunin IV. Non-coding RNA. Hum Mol Genet. 2006; 15:R17-R29. [PubMed: 16651366]

26. Viswanathan SR, Powers JT, Einhorn W, Hoshida Y, Ng TL, Toffanin S, et al. Lin28 promotes transformation and is associated with advanced human malignancies. Nat Genet. 2009; 41:843858. [PubMed: 19483683]

27. Cao D, Allan RW, Cheng L, Peng Y, Guo CC, Dahiya N, et al. RNA-binding protein LIN28 is a marker for testicular germ cell tumors. Hum Pathol. 2011; 42:710-718. [PubMed: 21288558]

28. Wang T, He Y, Zhu Y, Chen M, Weng M, Yang C, et al. Comparison of the expression and function of Lin28A and Lin28B in colon cancer. Oncotarget. 2016; 7:79605-79616. [PubMed: 27793004]

29. Salmena L, Poliseno L, Tay Y, Kats L, Pandolfi PP. A ceRNA hypothesis: the Rosetta Stone of a hidden RNA language. Cell. 2011; 146:353-368. [PubMed: 21802130]

30. Song YX, Sun JX, Zhao JH, Yang YC, Shi JX, Wu ZH, et al. Non-coding RNAs participate in the regulatory network of CLDN4 via ceRNA mediatedmiRNA evasion. Nat Commun. 2017; 8:289302. [PubMed: 28819095] 
31. Ma MZ, Zhang Y, Weng MZ, Wang SH, Hu Y, Hou ZY, et al. Long Noncoding RNA GCASPC, a Target of miR-17-3p, Negatively Regulates PyruvateCarboxylase-Dependent Cell Proliferation in Gallbladder Cancer. Cancer Res. 2016; 76:5361-5371. [PubMed: 27450454]

32. Lu W, Wang J, Yang G, Yu N, Huang Z, Xu H, et al. Posttranscriptional regulation of Galectin-3 by miR-128 contributes to colorectalcancer progression. Oncotarget. 2017; 8:15242-15351. [PubMed: 28146425]

33. Powers JT, Tsanov KM, Pearson DS, Roels F, Spina CS, Ebright R, et al. Multiple mechanisms disrupt the let-7 microRNA family in neuroblastoma. Nature. 2016; 535:246-251. [PubMed: 27383785]

34. Gokbuget D, Pereira JA, Bachofner S, Marchais A, Ciaudo C, Stoffel M, et al. The Lin28/let-7 axis is critical for myelination in the peripheral nervoussystem. Nat Commun. 2015; 6:8584-8593. [PubMed: 26466203]

35. Cho SW, Xu J, Sun R, Mumbach MR, Carter AC, Chen YG, et al. Promoter of lncRNA Gene PVT1 Is a Tumor-Suppressor DNA Boundary Element. Cell. 2018; 173:1398-1412. [PubMed: 29731168]

36. Di CS, Zhang F, Sancho A, Li S, Aguilo F, Sun Y, et al. RBM5-AS1 Is Critical for Self-Renewal of Colon Cancer Stem-like Cells. Cancer Res. 2016; 76:5615-5627. [PubMed: 27520449]

37. He F, Chen H, Yang P, Wu Q, Zhang T, Wang C, et al. Gankyrin sustains PI3K/GSK-3beta/betacatenin signal activation and promotescolorectal cancer aggressiveness and progression. Oncotarget. 2016; 7:81156-1171. [PubMed: 27835604]

38. He F, Peng F, Xia X, Zhao C, Luo Q, Guan W, et al. MiR-135a promotes renal fibrosis in diabetic nephropathy by regulating TRPC1. Diabetologia. 2014; 57:1726-1736. [PubMed: 24908566] 

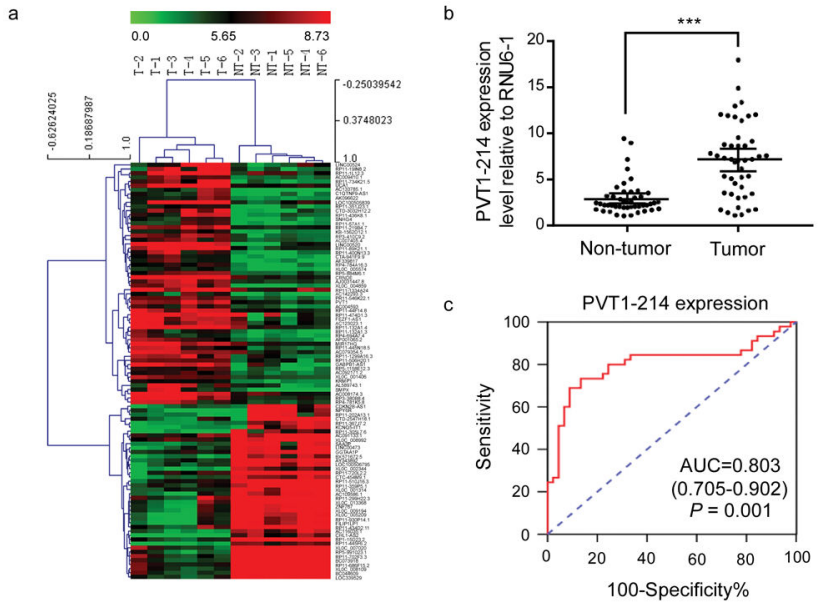

d
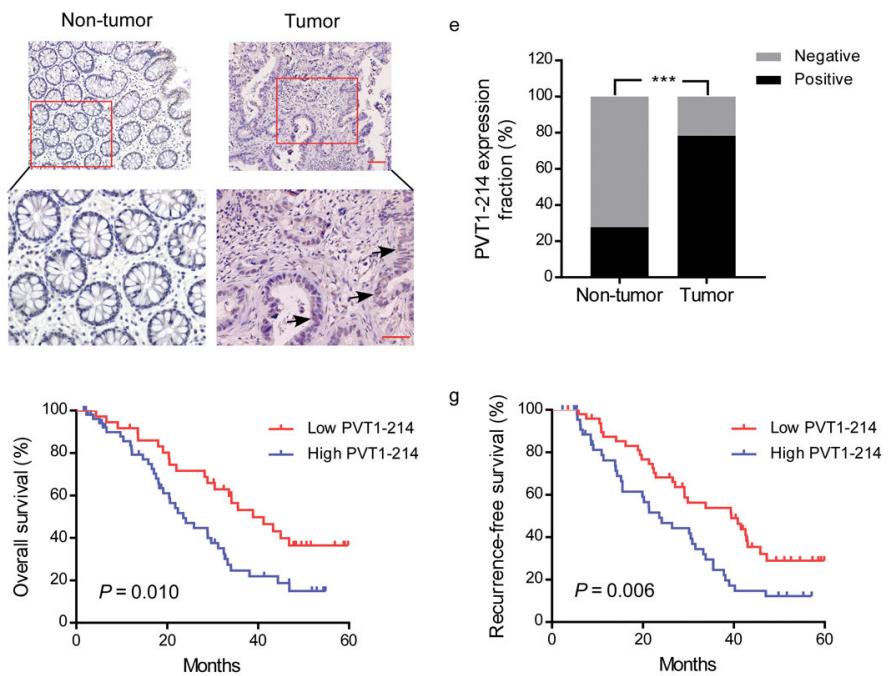

Figure 1. PVT1-214 is upregulated in human colorectal cancer (CRC) tissues and associated with poor prognosis

(a) The hierarchical clustering heat map of the top 100 lncRNAs differentially expressed between CRC samples (T, tumor) and paired non-tumor (NT) samples ( $>2$-fold; $P<0.05$ ).

(b) PVT1-214 expression was validated by qRT-PCR in CRC tumor samples and paired adjacent normal tissues (cohort $1, \mathrm{n}=45$ ). ***, $P<0.001$. (c) ROC curve analysis for the prediction of CRC using qRT-PCR-detected PVT1-214 expression. (d, e) Representative images of PVT1-214 expression were showed by in situ hybridization (ISH) (d), and quantitative analysis of $P V T 1-214$ expression detected by ISH (e). Scale bar $=50 \mu \mathrm{m}$. ***, $P$ $<0.001$. (f, g) Kaplan-Meier analysis of correlation between the PVT1-214 levels and overall survival (f) and recurrence-free survival (g) of CRC patients. 

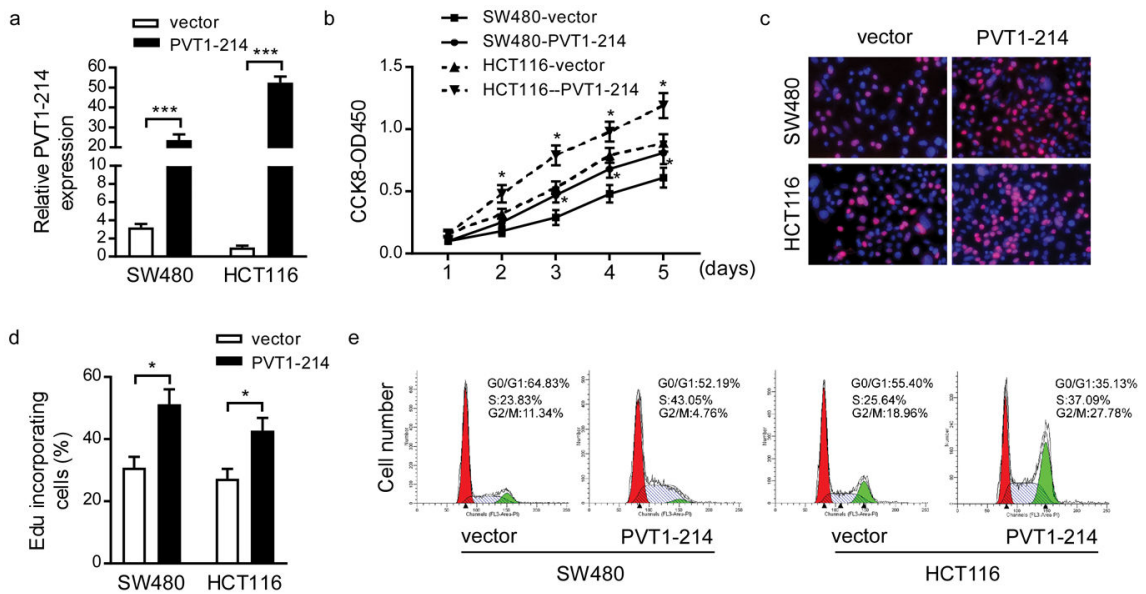

$f$
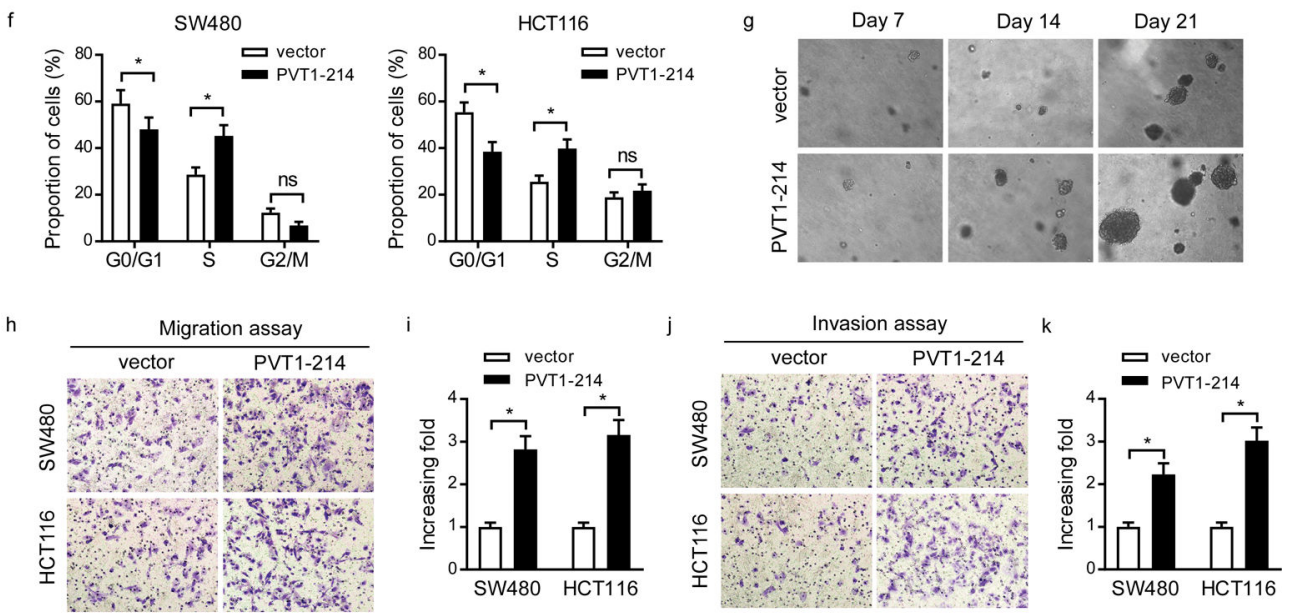

Figure 2. PVT1-214 promotes cell proliferation, stem-like properties, and metastasis of CRC cells in vitro

(a) qRT-PCR results showing the efficiency of PVT1-214 expression in stable PVT1-214 overexpressing SW480 and HCT116 cells. Data are the mean \pm SEM. $* * *, P<0.001$. (b) Graph depicting cell proliferation rates by CCK-8 assay. ${ }^{*}, P<0.05$. (c, d) Cellular proliferation was estimated from immunofluorescence staining with EdU (c) and the percentage of EdU-positive nuclei was quantified (d). ${ }^{*}, P<0.05$. (e, f) FACS analysis showing the phases of the cell cycle (e) and the percentage of cells in each cell-cycle (f). Data are the mean \pm SEM. ns, no significant. ${ }^{*}, P<0.05$. (g) Representative images of spheres formed from $P V T 1-214$ overexpressing cells and control cells, showing that PVT1-214 enhances the acquisition of stem cell like properties of SW480 cells. (h, i) Representative images (h) and quantification of the amount of migratory SW480 and HCT116 cells with or without exogenous PVT1-214 expression (i) per high-power field. (j, k) Representative images (j) and the quantification of invasive cells (k) per high-power field following overexpression of PVT1-214 in SW480 and HCT116 cells. The data are representative of three independent experiments. * $P<0.05$. 
a
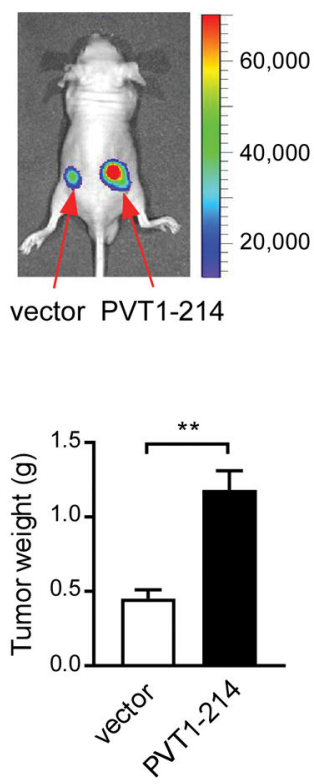

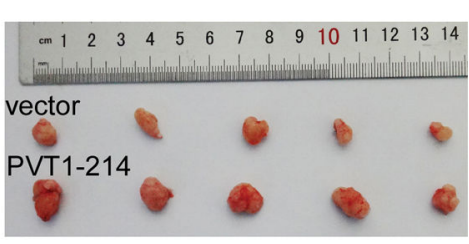

b

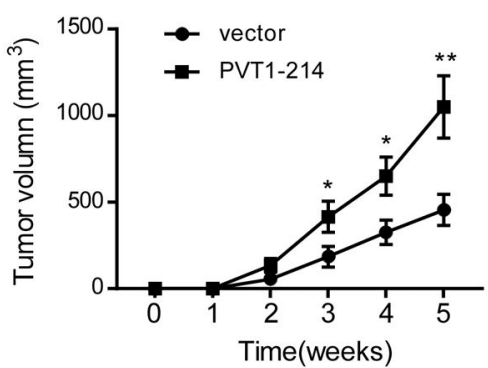

d

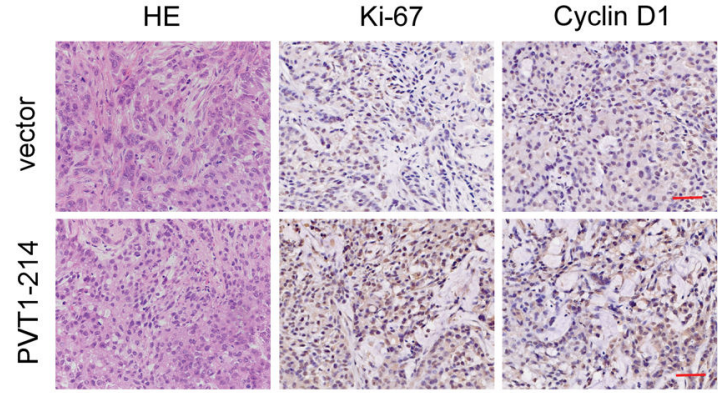

e

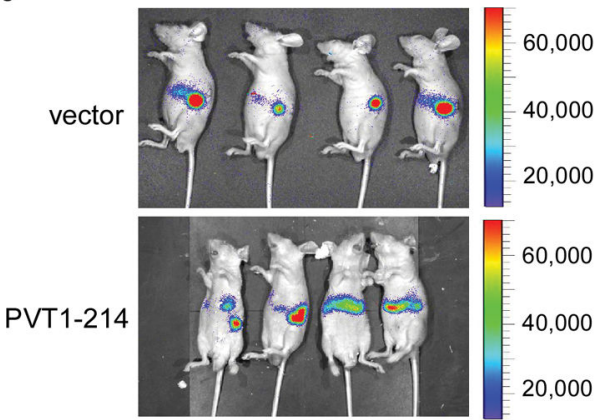

f

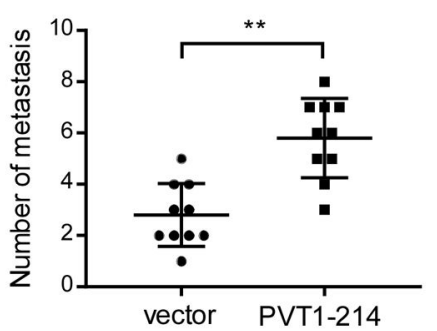

Figure 3. PVT1-214 accelerates the growth and metastasis of CRC cells in vivo

(a) Xenograft model in nude mice ( $n=5$ /group) viewed with the IVIS Imaging System. Left, representative tumor-bearing image. Right, tumors were photographed from each group. (b, c) Tumor growth curves (b) and tumor weights (c) was measured and calculated according to the Materials and Methods. Data represents the mean \pm SEM. ${ }^{*}, P<0.05$. ${ }^{*}, P$ $<0.01$. (d) Representative images of H\&E staining, and IHC staining for Ki67 and Cyclin D1. Scale bar $=50 \mu \mathrm{m}$. (e) Bioluminescent imaging of PVT1-214-overexpressing and luciferase expressing control HCT116 cells transplanted in BALB/c-nude mice. (f) Number of metastatic foci in liver were counted. Data are shown as mean \pm SEM. $* *, P<0.01$. 
a
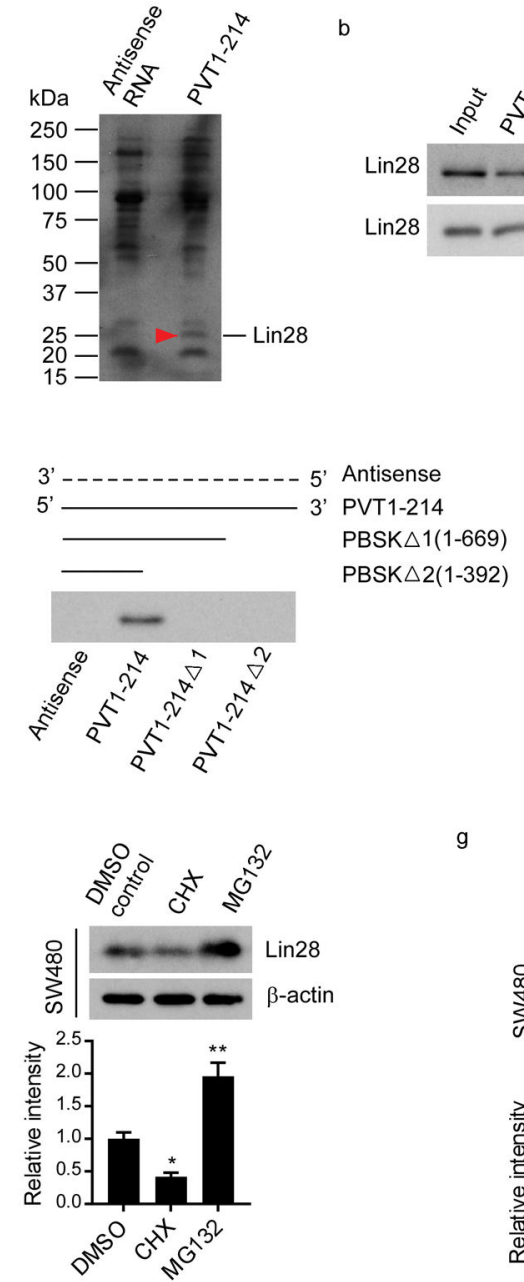

$b$

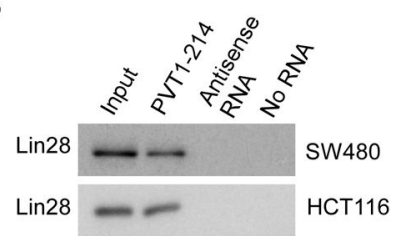

c

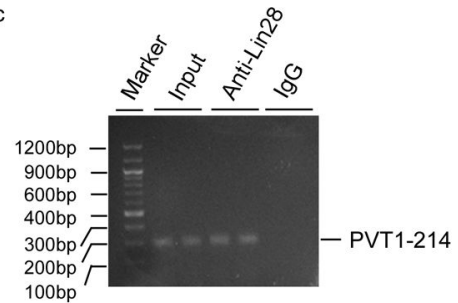

e
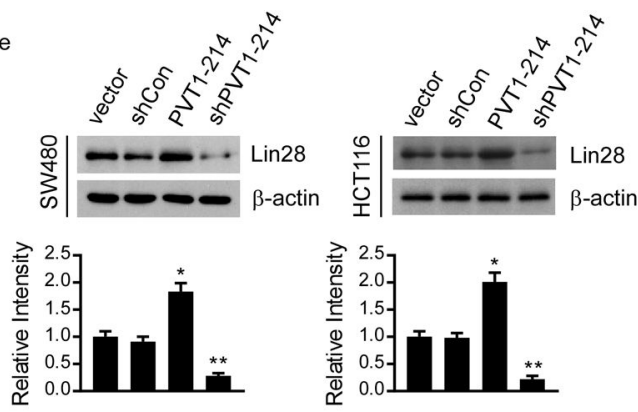

inl.
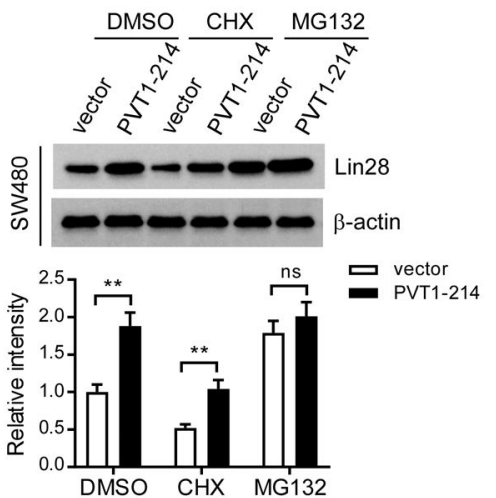

Figure 4. PVT1-214 binds to Lin28 protein and increases its protein expression by enhancing its stability

(a) Silver staining SDS-PAGE gel of electrophoretically separated proteins

immunoprecipitated with PVT1-214 and its antisense RNA in SW480 cells. Arrows indicate the regions of the gel selected to mass spectrometry using liquid chromatography dual mass spectrometry. (b) RNA pull-down assay was performed in SW480 and HCT116 cells using biotinylated $P V T 1-214$ or antisense RNA probe transcribed in vitro and detected by western blots. (c) RIP assays were performed in SW480 cells using Lin28 antibody to detect PVT1-214 RNA enrichment in immunoprecipitated complex. IgG is negative control. (d) RNA pull-down was performed using different $P V T 1-214$ deletion probes (top) and the enriched protein extract was detected with western blots (bottom) in SW480 cells. (e) Western blot analysis of Lin28 protein expression based PVT1-214 overexpressing or knockdown in SW480 and HCT116 cells. (f) Comparison and quantification of Lin28 protein expression in SW480 cells in the presence of the protein synthesis inhibitor cycloheximide (CHX) or the proteasome inhibitor MG-132. (g) Stably overexpressing PVT1-214 or vector cells were treated with CHX and MG132, and the Lin28 protein expression was measured by western blots (top) and quantified by densitometry (bottom). Data are shown as the mean \pm SEM $(\mathrm{n}=3)$. ns, no significant. *, $P<0.05$. **, $P<0.01$. 

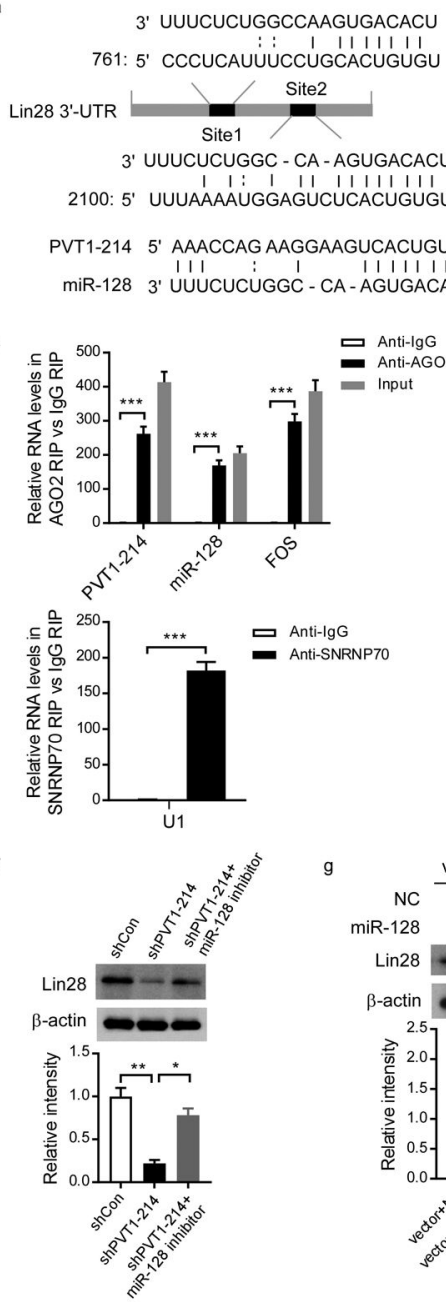

g b

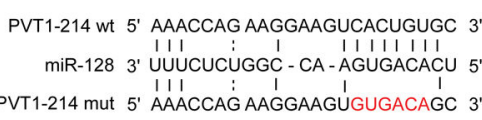
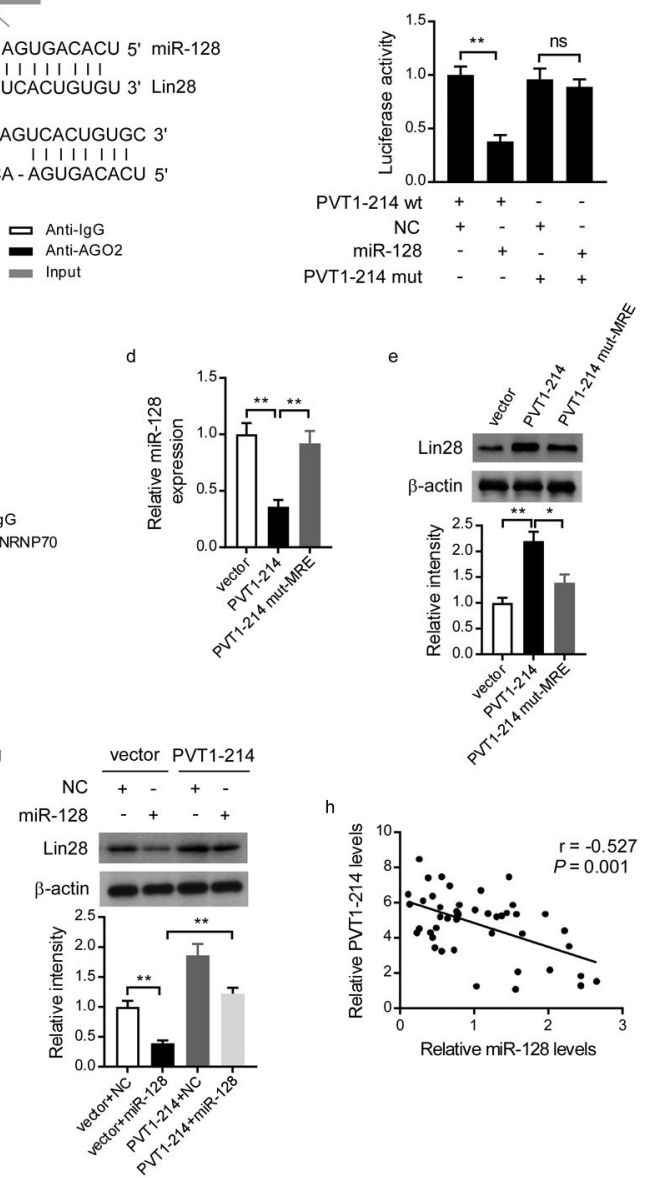

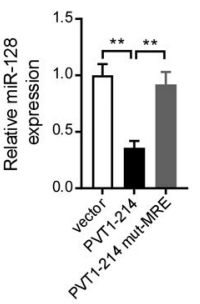

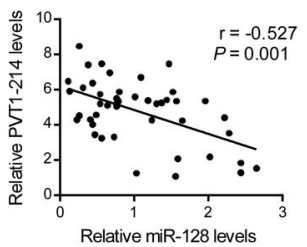

Figure 5. PVT1-214 acts as a molecular sponge for miR-128, and controls the miR-128 target, Lin28

(a) The putative miR-128 binding sites with the PVT1-214 sequence and Lin283'-UTR were showed. (b) Luciferase reporter gene assays were used to evaluate the interaction between miR-128 and PVT1-214. (c) RIP with anti-Ago2. Top: levels of PVT1-214, miR-128, and FOS RNA were enriched in Ago2 antibody compared to IgG immunoprecipitates. Bottom: the $U 1$ snRNA enriched in SNRNP70 antibody compared to IgG immunoprecipitates. Data represents the mean \pm SEM $(\mathrm{n}=3)$. ***, $P<0.001$. (d) Relative expression of miR-128 in PVT1-214 or PVT1-214 mutation of the putative miRNA recognition element (MRE) overexpressed-HCT116 cells. Data represents the mean \pm SEM from 3 independent experiments. **, $P<0.01$. (e) Western blotting confirmed Lin28 protein expression in indicated cells. $\beta$-actin was used as a loading control (top panel). The quantitative analysis of western blotting (bottom panel). Data are shown as mean \pm SEM (n $=3$ ). * $P<0.05$. **, $P<0.01$. (f) The Lin28 protein expression in $P V T 1-214$ knockdownHCT116 cells with or without miR-128 inhibitor (top panel). The quantitative analysis of western blots (bottom panel). (g) Western blotting analysis of Lin28 protein expression following expression of an empty vector or PVT1-214, and treatment with miRNA negative control or miR-128 mimics (top panel). The quantitative analysis of western blots (bottom 
panel). Data are shown as mean \pm SEM $(\mathrm{n}=3) . *, P<0.05$. **, $P<0.01$. (h) Bivariate correlation analysis of the association between $P V T 1-214$ expression and miR-128 levels. 


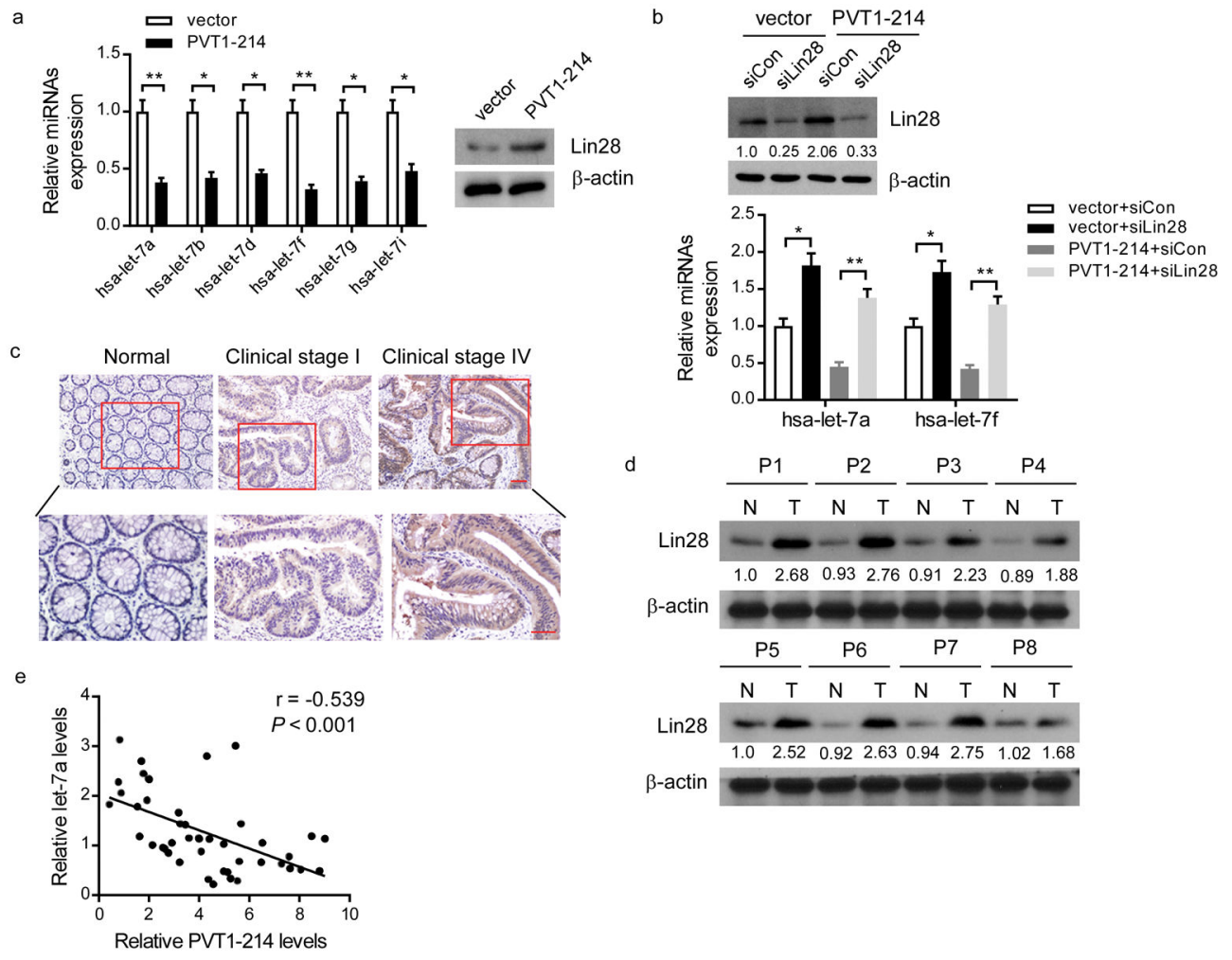

Figure 6. Up-regulation of $\operatorname{Lin} 28$ is necessary for $P V T 1-214$-induced let-7 repression (a) Real-time PCR analysis of different let-7 family members expression in SW480 cells stably expressing a control vector or PVT1-214 (left panel). Western blot of Lin28 expression in indicated cells (right panel). (b) Western blotting analysis of Lin28 protein expression in SW480 cells stably expressing an empty vector or PVT1-214, transfected with control or Lin 28 siRNAs (upper panel). Relative expression of let-7a and let-7f in indicated cells (bottom panel). Data are shown as mean \pm SEM $(\mathrm{n}=3)$. *,$P<0.05$. **, $P<0.01$. (c) IHC staining for human CRC tumor samples and normal colorectal tissues. Scale bars, 50 $\mu \mathrm{m}$. (d) Western blotting of Lin28 protein expression in eight pairs of surgically resected fresh CRC tissues. $\beta$-actin acted as the loading control. (e) Regression analysis illustrating a negative correlation between PVT1-214 and let-7a expression levels in CRC tissues. 

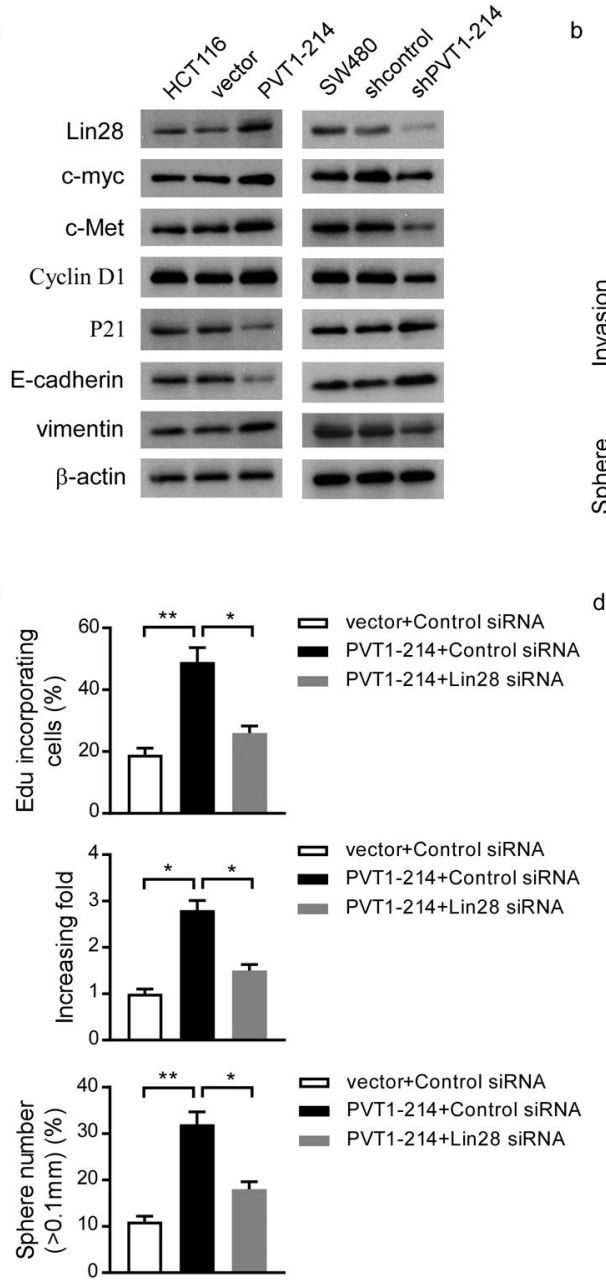

b

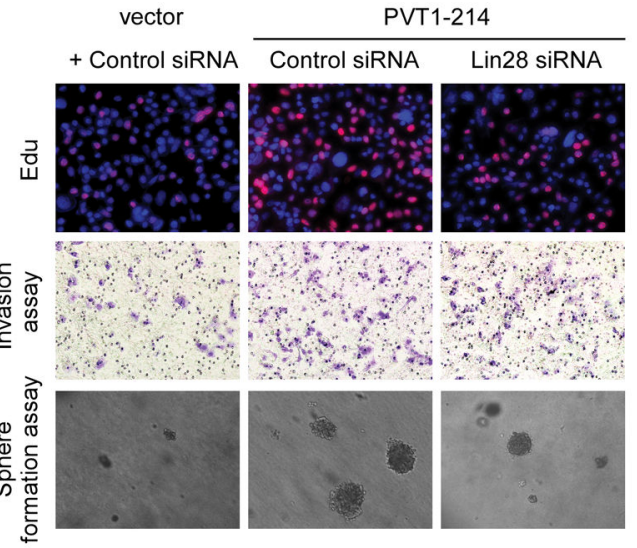

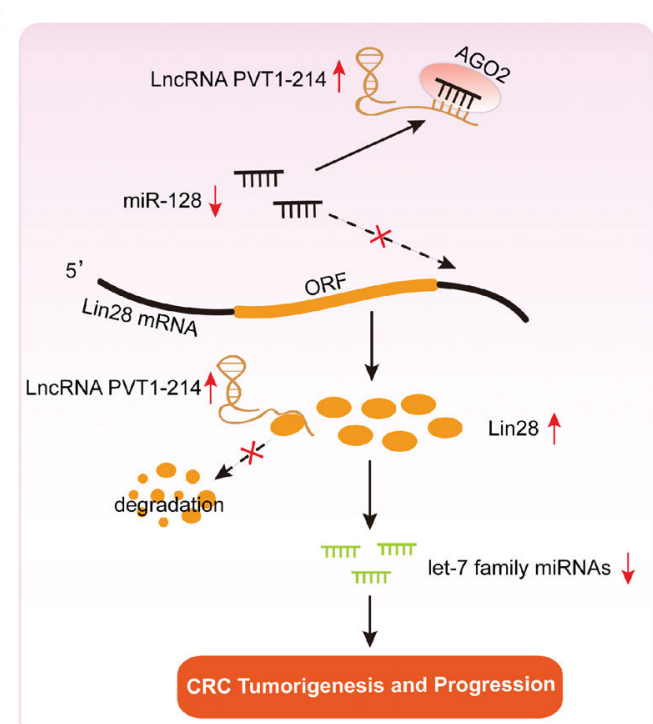

Figure 7. The biological function of PVT1-214 is dependent on of Lin28

(a) Western blot of indicated proteins in SW480 cells stably expressing a control vector or PVT1-214, or PVT1-214 shRNAs. $\beta$-actin was used as a loading control. (b, c) Lin28 siRNA and control siRNA were transfected into PVT1-214-overexpressing SW480 cells, and cell proliferation was detected with $\operatorname{EdU~(b,~c~top).~Cell~invasion~was~assessed~by~}$ Transwell assay with Matrigel (b, c middle), and cell stem-like properties were analyzed by the sphere formation assay $(\mathbf{b}, \mathbf{c}$ bottom). Data are shown as mean $\pm \operatorname{SEM}(\mathrm{n}=3) . *, P<$ 0.05 . **, $P<0.01$. (d) Schematic model schematic depicting the role of $P V T 1-214$ in CRC pathogenesis. 


\section{로을}

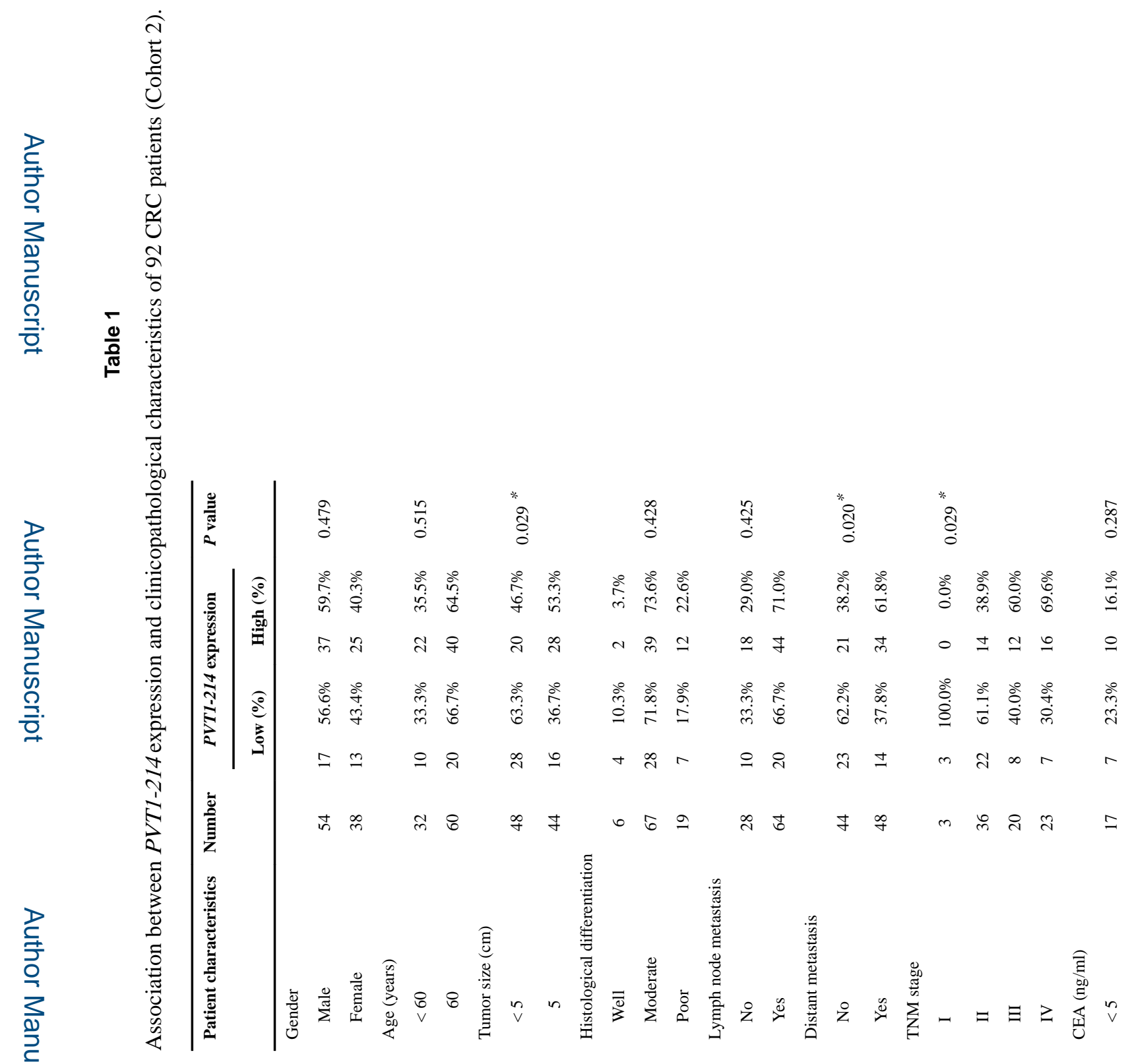




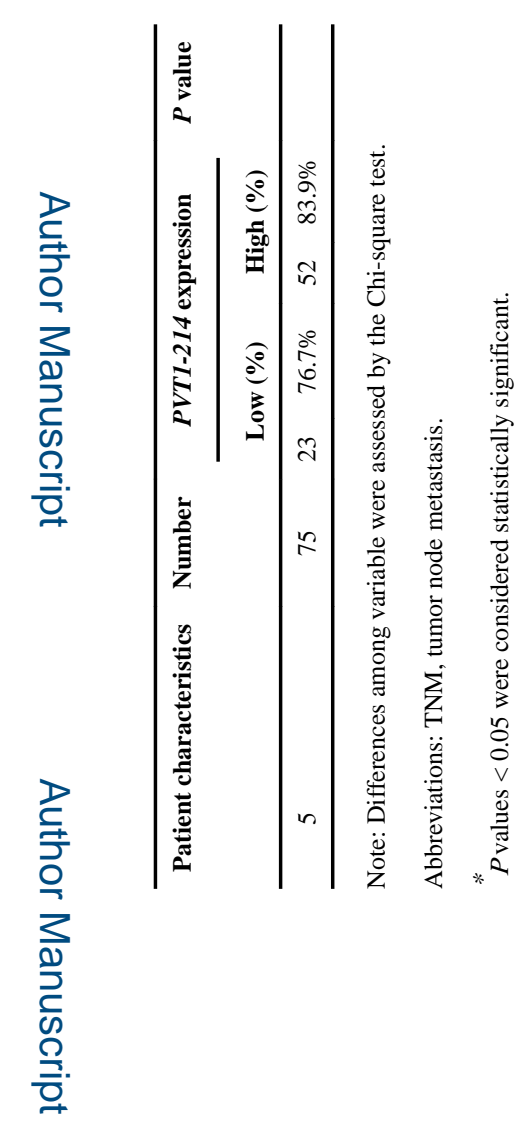

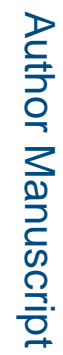

로을 